\title{
Clinical significance of smell and taste dysfunction and other related factors in COVID-19
}

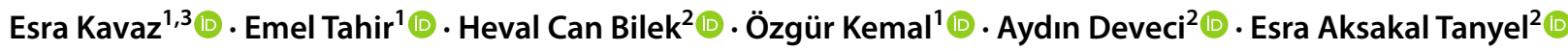

Received: 21 August 2020 / Accepted: 16 November 2020 / Published online: 1 January 2021

(c) Springer-Verlag GmbH Germany, part of Springer Nature 2021

\begin{abstract}
Purpose The objective of this study is to evaluate smell and taste dysfunction (STD) in coronavirus disease 2019 (COVID19) positive and negative patients, and to assess the factors associated with STD in COVID-19 positive patients.

Methods Patients who had been tested with the real-time reverse transcriptase-polymerase chain reaction (RT-PCR) for COVID-19 were identified, and according to the RT-PCR test results, patients were separated into Positive and Negative Groups. A telephone-based assessment was applied to both groups using the American Academy of Otolaryngology-Head and Neck Surgery Anosmia Reporting Tool. Patients in Positive Group were also asked to rate STD, nasal breathing, and anxiety in three different time periods (pre-/during-/post-COVID) using the visual analog scale (VAS).

Results A total of 53 COVID-19 positive and 51 negative patients completed the surveys. STD was eightfold more frequent (OR 8.19; CI 95\% 3.22-20.84) in the Positive Group. Of the 53 COVID-19 positive patients, 32 reported STD and 21 did not. 'Ground-glass appearance' on chest-computed tomography was more frequent and median lymphocyte count was significantly lower in COVID-19 positive patients with STD. During-COVID STD and nasal breathing VAS scores were significantly lower than the pre- and post-COVID scores. During-COVID STD scores were significantly correlated with anxiety scores (Spearman's rho-0.404, $p=0.022$ ) but not correlated with nasal breathing scores.

Conclusion STD may be related to increased inflammatory response as well as damage of olfactory neuronal pathway or non-neuronal olfactory mucosa. Understanding the exact cause of chemosensory impairment in COVID-19 can be helpful in explaining the pathophysiology of the disease.
\end{abstract}

Keywords COVID-19 $\cdot$ SARS-CoV-2 O Olfactory dysfunction $\cdot$ Anosmia $\cdot$ Visual analog scale

Electronic supplementary material The online version of this article (https://doi.org/10.1007/s00405-020-06503-9) contains supplementary material, which is available to authorized users.

\section{Introduction}

The coronavirus disease 2019 (COVID-19) caused by Severe Acute Respiratory Syndrome Coronavirus 2 (SARS-CoV-2) started in December 2019 in Wuhan (China) and the World Health Organization (WHO) declared COVID-19 as a pandemic on March 1, 2020 [1-3]. According to early reports,

Esra Aksakal Tanyel etanyel@omu.edu.tr

1 Department of Otorhinolaryngology and Head and Neck Surgery, Ondokuz Mayıs University School of Medicine, Samsun, Turkey

2 Department of Infectious Diseases, Ondokuz Mayis University School of Medicine, Samsun, Turkey

3 Present Address: Ondokuz Mayıs Üniversitesi Tıp Fakültesi KBB Anabilim Dalı, Körfez Atakum Samsun, Turkey 
the most common symptoms were fever, cough, dyspnea, myalgia, arthralgia, headache, diarrhea, rhinorrhea, and sore throat $[4,5]$. However, during the spread of the outbreak in Italy, Iran, and China, smell and taste dysfunctions (STD) have been reported by many clinicians [6-8]. The American Academy of Otolaryngology-Head and Neck Surgery (AAO-HNS) developed an 'Anosmia Reporting Tool' on March 26, 2020 [9]. It was reported that $73 \%$ of 237 patients had olfactory dysfunction in a validation study of the Anosmia Reporting Tool [10]. This anosmia reporting tool has enabled the collection of data in a standardized form during the clinical course of COVID-19. In a multicenter European study, $85.6 \%$ and $88.0 \%$ of 417 patients reported olfactory and gustatory dysfunctions, respectively [11]. After these developments, smell/taste loss was accepted as a symptom of COVID-19 by the European Centre for Disease Prevention and Control, the World Health Organization and patients with STD were identified as 'possible cases' [12, 13]. As olfactory and gustatory dysfunctions have become a noteworthy symptom of COVID-19, it has become necessary to study this subject to elucidate the pathophysiology of the disease. Currently, real-time reverse transcriptasepolymerase chain reaction (RT-PCR) is the most commonly used diagnostic tool to confirm the diagnosis of COVID-19.

The objective of this study is to evaluate STD in COVID19 positive and negative patients, and to assess the factors associated with STD in COVID-19 positive patients. Furthermore, this study evaluated the change of smell/taste function and nasal breathing in different periods of disease in COVID-19 patients with and without STD.

\section{Materials and methods}

\section{Patients and clinical outcomes}

This retrospective survey study was conducted at Ondokuz Mayıs University Hospital, Samsun, Turkey. Approval for the study was granted by the Institutional Ethics Committee (OMUKAEK 2020/349) and the study was conducted in accordance with the Declaration of Helsinki. Informed verbal consent was obtained from all the participants.

A total of 210 patients who were identified as 'possible cases' because of symptoms like fever, cough, shortness of breath, sore throat, headache, myalgia, diarrhea, and STD or who had close contact with COVID-19 positive patients even if asymptomatic, had been tested by RT-PCR for SARS-CoV-2 on nasopharyngeal swab from March 10 to June 5, 2020 and they were retrospectively identified from the records of Ondokuz Mayıs University Hospital.

Our study included patients who were tested because of symptoms matching the positive case definition, aged older than 18 , and who were native Turkish speakers. Pregnant women, patients tested for screening (asymptomatic people who had close contact with COVID-19 patients), patients who had remained in an intensive care unit, patients who were unable to complete the survey because of communicative or neurophysiological impairments, and patients reporting STD prior to disease were not included in the study. In the center where the study was conducted, RT-PCR negative patients are asked to apply for a retest in case of an increase in their complaints. RT-PCR negative patients with progression of their symptoms even if the second test results were negative, and patients who were considered positive according to their radiological and clinical findings were not included in the study to exclude 'false-negative' results.

Based on the abovementioned exclusion criteria, 19 of 72 RT-PCR positive patients and 87 of 138 RT-PCR negative patients were not included in study. Fifty-three RT-PCR positive patients and 51 RT-PCR negative patients constituted the Positive (Group P) and Negative Groups (Group $\mathrm{N}$ ), respectively. The demographic features of the patients (age, gender, and comorbidities), complete blood count results (lymphocyte count, neutrophil/lymphocyte ratio), C-reactive protein (CRP) level, radiological results (presence of ground-glass appearance on chest $\mathrm{CT}$ ) were retrieved from the medical records.

\section{Smell and taste outcomes}

All eligible patients were contacted by telephone and if the patient fulfilled the inclusion criteria, the questionnaires were completed with the assistance of the researchers. The AAO-HNS Anosmia Reporting Tool [9] was administered to both RT-PCR positive and negative patients. This tool includes 17 items related to demographic features (age, gender, and country), COVID-19 test status (positive, negative, presumed positive, and pending), risk factors for COVID19 infection (healthcare workers, the first responder, close contact with a confirmed case, homeless, congregate living, and travel to known areas with widespread community transmission), comorbidities, timing of STD (before/after diagnosis), symptoms, COVID-19 infection status, STD status (resolved/not resolved).

In addition to questions in AAO-HNS Anosmia Reporting Tool, patients with STD in Group P were asked questions about the onset of STD (progressive/sudden), duration of STD (longer than 1 week/less than 1 week), timing of STD (pre-sampling/after sampling), 'knowing about STD from media' (yes/no), smell disorder type (anosmia/hypos$\mathrm{mia} /$ parosmia) and taste disorder type (ageusia/hypogeusia/ dysgeusia).

Patients in Group P were asked to evaluate smell/taste functions (0: total loss of smell/taste, 10: excellent sense of smell/taste) and nasal breathing (0: complete blockage, 10 : excellent nasal breathing) using a visual analog scale (VAS) 
in 3 time periods (pre-COVID, during-COVID, and postCOVID). The time expressed as 'pre-COVID' was determined as the period before the pandemic. The term 'duringCOVID' was determined as during the disease. The term 'post-COVID' refers to 'four weeks after a 5 day treatment regimen administered to all patients following the diagnosis'. In addition, the anxiety status of the patients related to permanent STD was graded as follows; 0: not anxious, 10: very anxious. The abovementioned surveys and measures were applied to patients 4 weeks after the end of the treatment and follow up in the Positive Group and 4 weeks after the sampling in the Negative Group.

\section{Statistical analysis}

Sample size was determined using G*Power (v.3.1.9.6) software. The effect size was calculated as 0.622 assuming the assessment was performed between two independent groups. This power analysis indicated that a total sample of 100 participants with two equally sized groups of 50 patients was necessary to achieve a power of 0.95 . Statistical Package for the Social Sciences for Windows (SPSS version 23.0; IBM Corp, Armonk, NY, USA) was used to perform the statistical analyses. Parametric tests were used when the variables were normally distributed according to the Shapiro-Wilk and Kolmogorov-Smirnov tests. Non-parametric tests were employed when the sample size was $<30$ and the variables were not normally distributed. When exploring the relationship between continuous variables, the independent samples $t$ test, Mann-Whitney $U$ test, Friedman test were used. Spearman correlation analysis was performed to investigate the relationship between the continuous variables. Categorical variables were evaluated using Pearson's chi-square $\left(\chi^{2}\right)$ test, Continuity correction, and Fisher's exact test. Unadjusted odds ratio (OR) with 95\% confidence intervals (CI 95\%) was calculated, with the RT-PCR test results considered as the independent variable. The level of statistical significance was set at $p<0.05$.

\section{Results}

The surveys were completed by 53 RT-PCR positive patients in Group P and 51 RT-PCR negative patients in Group N. The mean age and female/male ratio were $42.7 \pm 14.1$ years and 29/24 in Group P, and 39.5 \pm 11.9 years and 24/24 in Group N, respectively. There was no significant difference in terms of age, gender, and comorbidities between the groups $(p>0.05)$. Regarding risk factors for COVID-19 infection, 'close contact with a confirmed case' was more frequent in Group $\mathrm{P}(p=0.002)$. The most common symptoms were fever $(54.7 \%)$ and cough (52.8\%) in Group P, and cough (56.9\%) and sore throat (56.9\%) in Group N. On admission,
$28.3 \%$ of the patients in Group P had STD, but not in any patient in Group $\mathrm{N}(p<0.001)$. In Group $\mathrm{P}$, the 'gastrointestinal system symptoms' were significantly more common than in Group N $(p=0.047)$. In Group N, the complaints of 'sore throat' and 'rhinorrhea' were more common than in Group $\mathrm{P}(p<0.001, p=0.030$, respectively). Based on radiological features, none of the patients had 'groundglass appearance' in Group N, whereas almost half of the patients in Group P had that appearance on chest CT scan $(p<0.001)$. The median lymphocyte count was significantly lower in Group P $(p=0.019)$. The demographic and clinical characteristics of the patients are summarized in Table 1.

The frequency of total (before and after sampling) STD was significantly higher in Group P (60.4\%) than in Group N (15.7\%) (Pearson's chi-square test; $p<0.001)$ (OR 8.19; CI 95\% 3.22-20.84). Of the 53 patients in Group P, 32 had STD and 21 patients did not report STD. The distribution of STD according to the groups is demonstrated in Table 2. Among COVID-19 positive patients, there was no statistical significance in terms of STD between female (37.5\% had STD) and male (39.6\% had STD) subjects ( $p=0.988)$.

In comparison of patients with and without STD in Group $\mathrm{P}$, the 'ground-glass appearance' on chest CT and 'myalgia' were more frequently encountered and the 'median lymphocyte count' was significantly lower in patients with STD ( $p=0.018, p=0.018, p=0.016$, respectively). The demographic and clinical characteristics of the patients with and without STD in Group P are summarized in Table 3. AAO-HNS Anosmia Reporting Tool items including demographic features, COVID-19 test status, risk factors for COVID-19 infection, comorbidities, and symptoms are summarized in Tables 1, 3 .

The VAS scores of smell/taste functions and nasal breathing were compared according to the time periods in patients with and without STD in Group P. In patients with STD in Group P, median STD VAS scores were 10.0, 2.0 and 10.0 in pre-COVID, during-COVID, and post-COVID periods, respectively. In pairwise comparisons, median during-COVID STD VAS score was significantly lower than median pre- and post-COVID STD VAS scores $(p<0.001)$. There was no difference between pre- and post-COVID STD VAS scores $(p=0.708)$. Pre-COVID, during-COVID, postCOVID STD VAS scores of patients without STD in Group $P$ were identical with a median value of 9.0.

Median nasal breathing VAS scores were 9.0, 7.0 and 9.0 in pre-COVID, during-COVID, and post-COVID periods, respectively, in patients with STD in Group P. In pairwise comparisons, median during-COVID nasal breathing VAS score was significantly lower than median pre- and post-COVID nasal breathing VAS scores $(p<0.005)$ and there was no difference between pre- and post-COVID nasal breathing scores $(p=0.901)$. In patients without STD, the pre-COVID, during-COVID, and post-COVID 
Table 1 Demographic and clinical features of patients in Group P and N

\begin{tabular}{|c|c|c|c|}
\hline & Group P $(n: 53)$ & Group $\mathrm{N}(n: 51)$ & $p$ \\
\hline \multicolumn{4}{|l|}{ Demographic features } \\
\hline Age $[$ mean $( \pm S D)]$ & $42.75( \pm 14.12)$ & $39.50( \pm 11.95)$ & $0.210^{\mathrm{a}}$ \\
\hline Female/male $(n)$ & $29 / 24$ & $27 / 24$ & $1.00^{\mathrm{e}}$ \\
\hline \multicolumn{4}{|l|}{ Comorbidities $[n(\%)]$} \\
\hline Hypertension & $8(15.1)$ & $4(7.8)$ & $0.395^{\mathrm{e}}$ \\
\hline Diabetes mellitus & $5(9.4)$ & $5(9.8)$ & $1.00^{\mathrm{c}}$ \\
\hline Chronic respiratory disease & $4(7.5)$ & $2(3.9)$ & $0.678^{\mathrm{c}}$ \\
\hline Cardiovascular disease & 0 & $2(3.9)$ & $0.238^{\mathrm{c}}$ \\
\hline Neurologic disease & $2(3.8)$ & 0 & $0.495^{\mathrm{c}}$ \\
\hline Smoking & $20(37.7)$ & $19(37.3)$ & $1.00^{\mathrm{e}}$ \\
\hline Sinusitis/allergic rhinitis & $14(26.4)$ & $6(11.8)$ & $0.100^{\mathrm{e}}$ \\
\hline Head trauma & $5(9.4)$ & $3(5.9)$ & $0.716^{\mathrm{c}}$ \\
\hline \multicolumn{4}{|l|}{ Risk factors for COVID-19 infection $[n(\%)]$} \\
\hline Health-care worker & $8(15.1)$ & $11(21.6)$ & $0.548^{\mathrm{e}}$ \\
\hline Close contact with a confirmed case & $17(32.1)$ & $3(5.9)$ & $0.002^{\mathrm{e}}$ \\
\hline Congregant living & $18(34)$ & $13(25.5)$ & $0.465^{\mathrm{e}}$ \\
\hline $\begin{array}{l}\text { Travel to known areas with widespread } \\
\text { Community transmission }\end{array}$ & $2(3.8)$ & $1(2.0)$ & $1.00^{\mathrm{c}}$ \\
\hline \multicolumn{4}{|c|}{ Complaints when the sample received from the patient $[n(\%)]$} \\
\hline Fever & $29(54.7)$ & $26(51.0)$ & $0.853^{\mathrm{e}}$ \\
\hline Cough & $28(52.8)$ & $29(56.9)$ & $0.829^{\mathrm{e}}$ \\
\hline Sore throat & $7(13.2)$ & $29(56.9)$ & $<0.001^{\mathrm{e}}$ \\
\hline Shortness of breath & $15(28.3)$ & $14(27.5)$ & $1.00^{\mathrm{e}}$ \\
\hline Headache & $16(30.2)$ & $18(35.3)$ & $0.730^{\mathrm{e}}$ \\
\hline Myalgia & $27(50.9)$ & $26(51.0)$ & $0.997^{\mathrm{b}}$ \\
\hline Malaise & $20(37.4)$ & $14(27.5)$ & $0.364^{\mathrm{e}}$ \\
\hline Nasal congestion & $6(11.3)$ & $7(13.7)$ & $0.941^{\mathrm{e}}$ \\
\hline Rhinorrhea & $1(1.9)$ & $7(13.7)$ & $0.030^{c}$ \\
\hline GIS symptoms & $17(32.1)$ & 7 (13.7) & $0.047^{\mathrm{e}}$ \\
\hline STD & $15(28.3)$ & $0(0)$ & $<0.001^{\mathrm{e}}$ \\
\hline \multicolumn{4}{|l|}{ Radiologic and labaratory findings } \\
\hline Ground-glass apperance on CT $[n(\%)]$ & $27(50.9)$ & $0(0)$ & $<0.001^{\mathrm{e}}$ \\
\hline CRP $[$ median $(\min -\max )]$ & $5.43(0.10-435.24)$ & $3.14(1.0-153.06)$ & $0.193^{\mathrm{d}}$ \\
\hline $\mathrm{L}[$ mean $( \pm \mathrm{SD})]$ & $1.69( \pm 0.99)$ & $2.13( \pm 0.89)$ & $0.019^{\mathrm{a}}$ \\
\hline $\mathrm{N} / \mathrm{L}[\operatorname{median}(\min -\max )]$ & $2.53(0.71-29.40)$ & $2.52(0.66-29.5)$ & $0.497^{\mathrm{d}}$ \\
\hline
\end{tabular}

Bold prints in ' $p$ ' column, indicate a significant difference between Group $\mathrm{P}$ and $\mathrm{N}$

$\%$ percentage, CRP C-reactive protein, COVID-19 coronavirus didease 2019, CT computed tomography, $F$ female, GIS gastrointestinal system, Group P COVID-19 positive patients, Group N COVID-19 negative patients, $L$ lymphocyte count, $M$ male, $\max$ maximum, $\min$ minimum, $N / L$ neutrophil/lymphoctye ratio, $S D$ standard deviation, STD smell/taste dysfunction

aindependent samples $t$ test

${ }^{\mathrm{b}}$ Pearson chi square

${ }^{c}$ Fisher exact test

${ }^{\mathrm{d}}$ Mann-Whitney $U$ test

${ }^{\mathrm{e}}$ Continuity correction nasal breathing VAS scores were identical with a median value of 9.0 as in the STD-VAS scores. Figure 1 summarized the change of STD and nasal breathing VAS scores in different periods for Group P.
In patients with STD in Group P, there was a positive correlation between the pre-COVID nasal breathing VAS score and the pre-COVID STD VAS score in correlation analysis evaluating the relationship between STD, nasal breathing, 
Table 2 Comparison of smell/ taste dysfunction between Group P and N

Table 3 Demographic and clinical characteristics of patients with and without smell/ taste dysfunction in Group P

\begin{tabular}{llllll}
\hline & $\begin{array}{l}\text { Group P } \\
{[n(\%)]}\end{array}$ & $\begin{array}{l}\text { Group N } \\
{[n(\%)]}\end{array}$ & $\begin{array}{l}\text { Total } \\
{[n(\%)]}\end{array}$ & OR (CI 95\%) & $p^{\text {a }}$ \\
\hline \multicolumn{2}{l}{ Smell/taste Dysfunction } & & & \\
Yes & $32(60.4)$ & $8(15.7)$ & $40(38.5)$ & $8.19(3.22-20.84)$ & $<\mathbf{0 . 0 0 1}$ \\
No & $21(39.6)$ & $43(84.3)$ & $64(61.5)$ & \\
Total $[n(\%)]$ & $53(50.9)$ & $51(49.0)$ & $104(100)$ & & \\
\hline
\end{tabular}

Bold prints in ' $p$ ' column, indicate a significant difference between groups

Group P COVID-19 positive patients, Group N COVID-19 negative patients, OR Odds ratio, CI confidence interval

${ }^{a}$ Continuity correction

\begin{tabular}{|c|c|c|c|}
\hline & With STD in Group P $(n: 32)$ & $\begin{array}{l}\text { Without STD in } \\
\text { Group P }(n: 21)\end{array}$ & $p$ \\
\hline \multicolumn{4}{|l|}{ Demographic features } \\
\hline Age $[$ mean $( \pm S D)]$ & $41.18( \pm 14.29)$ & $45.14( \pm 13.85)$ & $0.323^{\mathrm{a}}$ \\
\hline $\mathrm{F} / \mathrm{M}(\mathrm{n})$ & $16 / 16$ & $13 / 8$ & $0.569^{\mathrm{e}}$ \\
\hline \multicolumn{4}{|l|}{ Comorbidities $[n(\%)]$} \\
\hline Hypertension & $6(18.8)$ & $2(9.5)$ & $0.455^{\mathrm{c}}$ \\
\hline Diabetes mellitus & $3(9.4)$ & $2(9.5)$ & $1.00^{\mathrm{c}}$ \\
\hline Chronic respiratory disease & $3(9.4)$ & $1(4.8)$ & $1.00^{\mathrm{c}}$ \\
\hline Neurologic disease & $1(3.1)$ & $1(4.8)$ & $1.00^{\mathrm{c}}$ \\
\hline Smoking & $10(31.3)$ & $10(47.6)$ & $0.361^{\mathrm{e}}$ \\
\hline Sinusitis/allergic rhinitis & $10(31.3)$ & $4(19)$ & $0.505^{\mathrm{e}}$ \\
\hline Head trauma & $3(9.4)$ & $2(9.5)$ & $1.00^{\mathrm{c}}$ \\
\hline \multicolumn{4}{|c|}{ Complaints when the sample received from the patient $[n(\%)]$} \\
\hline Fever & $20(62.5)$ & $9(42.9)$ & $0.261^{\mathrm{e}}$ \\
\hline Cough & $20(62.5)$ & $8(38.1)$ & $0.144^{\mathrm{e}}$ \\
\hline Sore throat & $4(12.5)$ & $3(14.3)$ & $1.00^{\mathrm{c}}$ \\
\hline Shortness of breath & $9(28.1)$ & $6(28.6)$ & $1.00^{\mathrm{e}}$ \\
\hline Headache & $12(37.5)$ & $4(19.0)$ & $0.260^{\mathrm{e}}$ \\
\hline Myalgia & $21(65.6)$ & $6(28.6)$ & $\mathbf{0 . 0 1 8}^{\mathrm{e}}$ \\
\hline Malaise & $12(37.5)$ & $8(38.1)$ & $1.00^{\mathrm{e}}$ \\
\hline Nasal obstruction & $5(15.6)$ & $1(4.8)$ & $0.384^{\mathrm{c}}$ \\
\hline Rhinorrhea & $1(3.1)$ & $0(0)$ & $1.00^{\mathrm{c}}$ \\
\hline GIS symptoms & $10(31.3)$ & $7(33.3)$ & $1.00^{\mathrm{e}}$ \\
\hline \multicolumn{4}{|l|}{ Radiologic and labaratory findings } \\
\hline Ground-glass apperance on CT $[n(\%)]$ & $21(65.6)$ & $6(28.6)$ & $0.018^{\mathrm{e}}$ \\
\hline CRP $[\operatorname{median}(\min -\max )]$ & $8.07(1.0-349.0)$ & $3.14(0.10-435.24)$ & $0.355^{\mathrm{d}}$ \\
\hline $\mathrm{L}[$ mean $( \pm \mathrm{SD})]$ & $1.43( \pm 0.85)$ & $2.10( \pm 1.08)$ & $0.016^{\mathrm{a}}$ \\
\hline $\mathrm{N} / \mathrm{L}[\operatorname{median}(\min -\max )]$ & $2.24(0.71-12.5)$ & $2.71(1.30-29.40)$ & $0.940^{\mathrm{d}}$ \\
\hline
\end{tabular}

Bold prints in ' $p$ ' column indicate a significant difference between with STD patients and without STD patients

$\%$ percentage, CRP C-reactive protein, COVID-19 coronavirus Didease 2019, CT computed tomography, $F$ female, GIS gastrointestinal system, Group $P$ COVID-19 positive patients, $L$ lymphocyte count, $M$ male, max maximum, $\min$ minimum, $N / L$ neutrophil/lymphoctye ratio, $S D$ standard deviation, $S T D$ smell/taste dysfunction

aindependent samples $t$ test

${ }^{\mathrm{b}}$ Pearson chi square

${ }^{c}$ Fisher exact test

${ }^{\mathrm{d}}$ Mann-Whitney $U$ test

${ }^{\mathrm{e}}$ continuity correction 

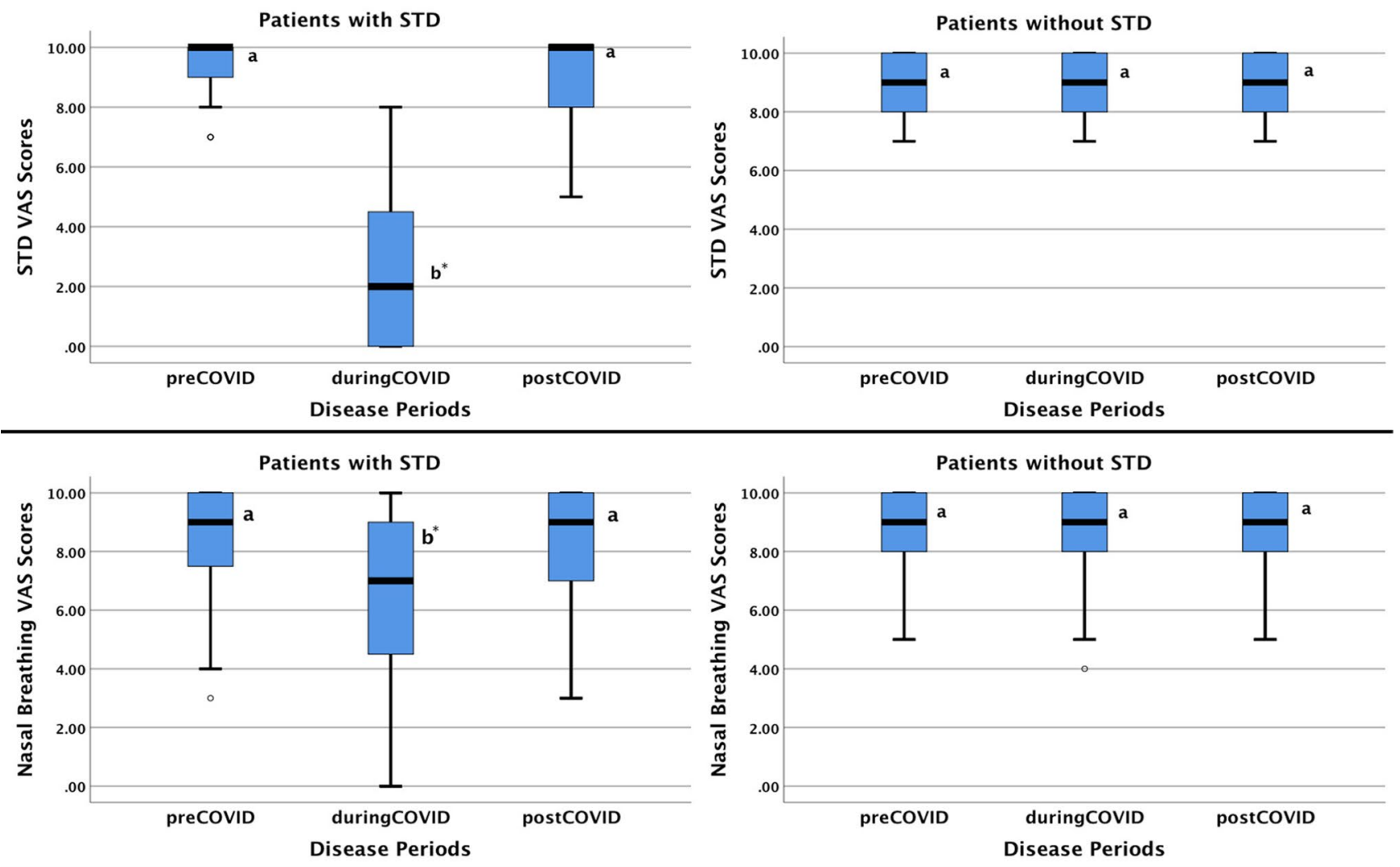

Fig. 1 Smell/taste dysfunction-related VAS scores (STD-VAS) and nasal breathing VAS scores (breathing VAS) of patients with and without STD in Group $\mathrm{P}$ in pre-COVID, during-COVID, and postCOVID periods. STD, smell/taste dysfunction; VAS, visual analog scale. Boxes indicate the first and third quartiles, and median obser-

and anxiety scores (Spearmans' rho $=0.406, p=0.021$ ). During the disease period, nasal breathing VAS score and STD VAS score were not significantly correlated. (Spearmans' rho:-0.064, $p=0.729$ ). The during-COVID STD-VAS score was only significantly correlated with the anxiety VAS score (Spearmans' rho $-0.404, p=0.022$ ). There was a positive correlation between the post-COVID nasal breathing VAS score and post-COVID STD VAS score (Spearmans' rho $=0.351, p=0.049)$. Figure 2 shows the correlation plot between STD, nasal breathing, and anxiety VAS scores.

Anosmia and hyposmia were significantly more common than parosmia in patients with STD in Group P $(p=0.003)$. Also, ageusia and hypogeusia were more common than dysgeusia in patients reported with taste dysfunction in Group $\mathrm{P}(p=0.004)$. For patients with STD in Group P, $47 \%$ of patients reported STD at the time of sampling and STD occurred during the course of the disease in $53 \%$ of patients $(\mathrm{p}=0.724)$. STD was resolved in less than 1 week in $75 \%$ of patients $(p=0.005)$. The onset of STD was predominantly progressive $(75 \%, p=0.005)$. Fifty-six percent of patients vations are denoted by a line in each box. Whisker caps indicate the minimum and maximum values. $\mathbf{a}, \mathbf{b}$ There is no difference between groups with the same letter in each box-plot. *Significantly different measurement after Friedman test with pairwise comparisons $(p<0.05)$

with STD had known about the association of STD and COVID-19 from media $(p=0.480)$. Figure 3 summarized the distribution of smell and taste disorder types, characteristics of STD such as timing, duration, and onset and the 'knowing about STD from media' among the patients with STD in Group P.

\section{Discussion}

When COVID-19 first emerged, fever, cough, and shortness of breath were reported as the predominant symptoms, as patients frequently complained about them. With the global spread of the disease, numerous clinicians around the world have reported smell/taste impairment as a symptom of the disease [6-8]. In the early phases of the pandemic, chemosensory function was evaluated using non-standardized self-reported questionnaires or subjective scales such as VAS [14-16]. AAO-HNS developed a COVID-19-specific Anosmia Reporting Tool to be able to 
Fig. 2 Correlation plot between STD, nasal breathing, and anxiety VAS scores in pre-COVID, during-COVID, post-COVID periods in patients with STD in Group P. Inside the cells; Spearman's rho coefficient was demonstrated. ${ }^{*} p<0.05$, $* * p<0.001$
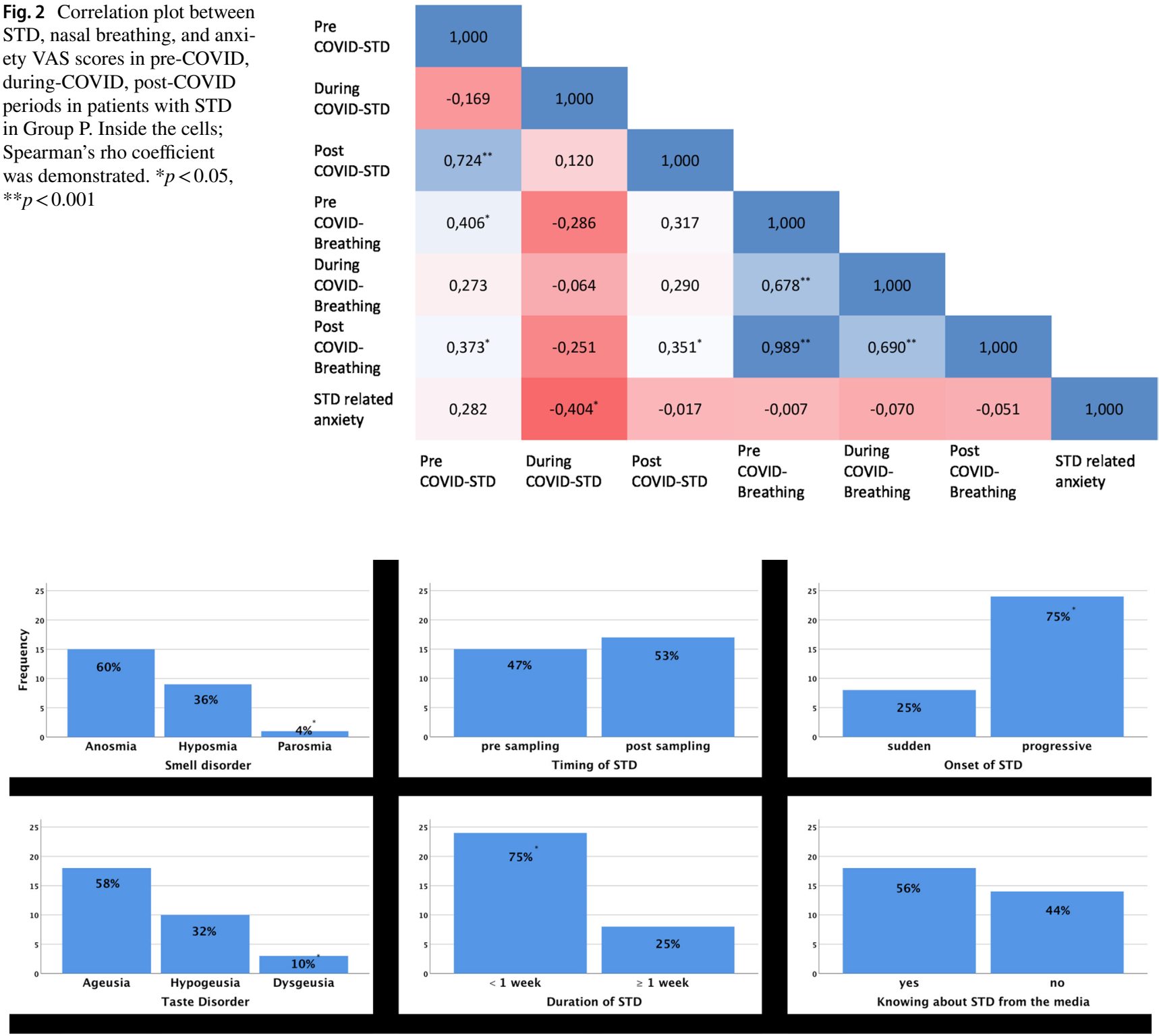

Fig. 3 Distribution of smell and taste disorder types, characteristics of STD such as timing, duration, and onset and the knowing about STD from media among the patients with STD in Group P. STD, smell/taste dysfunction. *Significantly different parameter (chi square test, $p<0.05$ )

clarify the chemosensory effects of the COVID-19 infection and standardize the patient-reported outcome measures. This tool includes 17 items related to smell/taste dysfunction as well as demographic features, risk factors, symptoms, and COVID-19 status [9]. In this study, a subjective VAS scale and a structured anosmia-specific tool, the AAO-HNS Anosmia Reporting Tool, were concomitantly used to enrich the results. According to the initial findings of this tool from the USA, olfactory dysfunction occurs at any time during the infection but tends to begin in the early course of the disease. In a validation study of the Anosmia Reporting Tool, $73 \%$ of 237 patients had olfactory dysfunction before the diagnosis and anosmia was the initial symptom in $26.6 \%$ of the positive cases [10]. In this study sample, $60.4 \%$ of the 53 RT-PCR positive patients reported STD. In accordance with other studies in the literature, there was a significant association between STD and COVID-19 since these chemosensory impairments were at least eightfold frequent (OR 8.19; CI 95\% 3.22-20.84) in COVID-19 positive patients.

Some studies have reported female or male domination in the development of chemosensory dysfunctions associated with gender-related differences in the inflammatory reaction process $[11,17,18]$. However, in the current study, no significant relationship was determined between gender and STD. 
Sayin et al. reported that most of the smell/taste dysfunctions were in hyposmia/hypogeusia forms and most patients recovered [19]. AAO-HNS Anosmia Reporting Tool was used in the current study to compare the results with current literature. In this study, olfactory dysfunction was mainly as anosmia and gustatory dysfunction was predominantly as ageusia, unlike the findings by Sayin et al.

Viral upper respiratory tract infections often cause a transient olfactory loss due to nasal congestion and rhinorrhea. However, in rare cases, a permanent olfactory loss may occur due to the viral involvement of the olfactory neuronal pathways [20]. In this study, $75 \%$ of the patients with STD reported a short course of sensory dysfunction as less than one week. In accordance with our findings, Leichen et al. reported an early recovery rate of $55 \%$ occurring in the first week of the disease [11]. Additionally, Meini et al. informed that most of the patients recovered from STD in 4 weeks [18]. Although this transient nature of the STD suggests that it may be due to nasal inflammation, many authors reported an increased frequency of smell/taste dysfunction which was more frequent than in an usual upper respiratory infection $[15,21]$. Barillari et al., analyzed the relationship between STD and otolaryngology-related symptoms such as nasal obstruction, rhinorrhea, and post-nasal drip and they found no association between nasal symptoms and STD [22]. Sinonasal manifestations of COVID-19 were studied by Mercante et al. with the Sino-Nasal Outcome Test 22 (SNOT22). As in our study, a telephone survey was applied and according to the findings, nasal obstruction was not frequently encountered at the onset of the disease [23]. Since the during-COVID STD and nasal breathing VAS scores in COVID-19 positive patients with STD were significantly lower compared to pre-COVID and post-COVID scores, we examined the correlation analysis to assess whether the STD was due to the nasal obstruction. There was no significant correlation between during-COVID STD and nasal breathing VAS scores. In other words, the severity of nasal blockage and chemosensory dysfunction had not been equally affected. This lack of correlation between nasal breathing and STD may be due to the fact that the virus causes neuronal damage in the olfactory pathway resulting in STD. Previously, olfactory dysfunction had been reported in other coronavirus infections such as SARS-CoV (the etiological agent of Severe Acute Respiratory Syndrome) [24]. In an animal study conducted in 2008, it was demonstrated that SARS-CoV can enter the brain via the olfactory bulb and causes neurological and chemosensory dysfunction [24]. Vaira et al. reported that the development of STD may be due the damage to the olfactory neuronal pathways by the virus [25]. On the other hand, Bran et al. hypothesized that inflammation of non-neuronal cells in the olfactory mucosa rather than neuronal damage causes STD, since the loss of smell and taste quickly recovers and other neurological symptoms are not frequent [26].

Recently, a new opinion has been proposed that an increased systemic inflammatory response is responsible in the pathophysiology of STD [27]. Oliviero et al. reported that CXCL10, a pro-inflammatory substance that causes pulmonary involvement, is also neurotoxic and may lead to chemosensory dysfunction [27]. Yan et al. have proposed that two forms of the infection may exist; nasal centric and pulmonary centric. They hypothesized that ambulatory cases have nasal-centric viral spread and they reported STD has been determined frequently in ambulatory cases [28]. Conversely, Meini et al. reported that chemosensory dysfunction was more frequent in patients requiring hospitalization because of respiratory failure [18]. Speth et al. noticed that the shortness of breath was more severe in patients experiencing olfactory dysfunction [29]. Although, in this study, there was no difference between patients with and without STD in Group P in terms of shortness of breath, 'groundglass appearance' was significantly more frequent in the patients with STD. In a recent meta-analysis, decreased lymphocyte count was related to an unfavorable prognosis [30]. Zhou et al. found that $80 \%$ of the patients, with pulmonary involvement documented by CT, had low lymphocyte counts [31]. Interestingly, in this cohort, 'groundglass appearance' and 'lower lymphocyte count' were more frequent in patients with STD. This fact supports the idea that pulmonary involvement and increased inflammatory response may be associated with chemosensory impairment. Abdullahi et al. reported that the interleukin- 6 and increased inflammatory response can be the cause of the myalgia in COVID-19 [32]. It is remarkable that myalgia was significantly higher in patients with STD in Group P in this study. Based on these studies and our findings, it may be speculated that increased systemic inflammation provokes chemosensory dysfunction. However, the pathophysiological and molecular mechanisms relating the chemo-sensitive disorders and inflammation should be clarified in future studies.

More than half of our patients had information about COVID-19 and the STD relationship. Although negative news and unfiltered information could negatively affect the mental health of people, news and social media had become more important because of curiosity about disease symptoms and lockdown [33, 34]. Bendau et al. reported that media exposure was positively correlated with anxiety specific to COVID-19 [34]. It would not be correct to say that mental health is affected only by the media. Speth et al. reported that STD was associated with depressed mood and anxiety in COVID-19 patients [29]. In the current study, a weak negative (rho $-0.404, p=0.022$ ) correlation was observed between the anxiety VAS scores and STD VAS scores during the disease. Although, chemosensory dysfunction may cause 
anxiety, neuronal degeneration due to infection may lead to psychological alterations. The correlation between anxiety and STD could be explained by two hypotheses. First, chemosensory dysfunction could cause anxiety and depression by decreasing the quality of life, whereas the other hypothesis suggests that chemosensory dysfunction and emotional disturbance could occur as a result of neuronal degeneration.

Despite the evolving literature on STD in COVID-19, the timing, duration, risk factors, and contribution of psychological factors have not yet been clarified. STD was observed to be highly prevalent in COVID-19 and frequently has a transient nature consistent with abovementioned literature. Previous studies were also extended by demonstrating that pulmonary involvement which manifests as 'ground-glass appearance' on radiological examination, myalgia and decreased lymphocyte count are associated with a higher risk of STD.

One of the limitations of the current study was the lack of objective examinations for olfactory/gustatory dysfunction, but these were intentionally avoided due to the high risk of transmission. Furthermore, as these are the findings of a single institution, there is a clear need for further more comprehensive studies on this subject.

STD should not be underestimated since it can be related to increased inflammatory response as well as pulmonary involvement. Olfactory neuronal degeneration or damage of nonneuronal olfactory mucosa may have a concomitant role in STD in patients with COVID-19. As chemosensory alterations can be a predictor of a positive RT-PCR result, future clinical and experimental research is needed to clarify the chemosensory impact of COVID-19.

Acknowledgements The authors would like to thank Prof. Dr. Sinan Atmaca for his peer review and linguistic support.

Author contributions Conceptualization: [EK, ET, HCB, AD, ÖK, EAT], Methodology: [EK, ET, HCB, ÖK], Formal analysis and investigation: [EK, ET, HCB, AD, ÖK, EAT], Writing —original draft preparation: [EK, ET, HCB, AD, ÖK, EAT], Writing-review and editing: [AD, ÖK, EAT], Resources: [EK, ET], Supervision: [AD, ÖK, EAT].

Funding This research received no specific grant from any profit or non-profit institution.

\section{Compliance with ethical standards}

Conflict of interest The authors declare there are no conflicts of interest-financial or otherwise-related to the material presented herein.

Ethics committee approval The ethic committee approval was received from the Ondokuz Mayıs University Ethics Committee (OMUKAEK 2020/349). All procedures performed in this study involving human participants were in accordance with the 1964 Declaration of Helsinki and its later amendments.

Consent to participate Verbal informed consent was obtained prior to the interview.
Consent for publication The participants have consented to the submission of their data to the journal.

Availability of data and material Available.

\section{References}

1. WHO Clinical management of COVID-19 (2020) https://www. who.int/publications/i/item/clinical-management-of-covid-19..

2. Wu D, Wu T, Liu Q, Yang Z (2020) The SARS-CoV-2 outbreak: What we know. Int J Infect Dis 94:44-48. https://doi. org/10.1016/j.ijid.2020.03.004

3. Mahase E (2020) Covid-19: WHO declares pandemic because of "alarming levels" of spread, severity, and inaction. Bmj-Brit Med J. https://doi.org/10.1136/bmj.m1036

4. Xu XW, Wu XX, Jiang XG, Xu KJ, Ying LJ, Ma CL, Li SB, Wang HY, Zhang S, Gao HN, Sheng JF, Cai HL, Qiu YQ, Li LJ (2020) Clinical findings in a group of patients infected with the 2019 novel coronavirus (SARS-Cov-2) outside of Wuhan, China: retrospective case series. BMJ 368:m606. https://doi.org/10.1136/ bmj.m606

5. Chen N, Zhou M, Dong X, Qu J, Gong F, Han Y, Qiu Y, Wang J, Liu Y, Wei Y, Xia J, Yu T, Zhang X, Zhang L (2020) Epidemiological and clinical characteristics of 99 cases of 2019 novel coronavirus pneumonia in Wuhan, China: a descriptive study. Lancet 395(10223):507-513. https://doi.org/10.1016/S0140 -6736(20)30211-7

6. Giacomelli A, Pezzati L, Conti F, Bernacchia D, Siano M, Oreni L (2020) Self-reported olfactory and taste disorders in SARSCoV-2 patients: a cross-sectional study. Clin Infect Dis. https:// doi.org/10.1093/cid/ciaa330 ([published online ahead of print March 26, 2020])

7. Bagheri SH, Asghari A, Farhadi M, Shamshiri AR, Kabir A, Kamrava SK, Jalessi M, Mohebbi A, Alizadeh R, Honarmand AA, Ghalehbaghi B, Salimi A, Dehghani Firouzabadi F (2020) Coincidence of COVID-19 epidemic and olfactory dysfunction outbreak in Iran. Med J Islam Repub Iran 34:62. https://doi.org/10.34171/ mjiri.34.62

8. Mao L, Jin H, Wang M, Hu Y, Chen S, He Q, Chang J, Hong C, Zhou Y, Wang D (2020) Neurologic manifestations of hospitalized patients with coronavirus disease 2019 in Wuhan. China JAMA neurology 77(6):683-690

9. American Academy of Otolaryngology-Head and Neck Surgery (2020) COVID-19 Anosmia Reporting Tool for Clinicians. https ://www.entnet.org/content/reporting-tool-patients-anosmia-relat ed-covid-19.

10. Kaye R, Chang CWD, Kazahaya K, Brereton J, Denneny JC 3rd (2020) COVID-19 anosmia reporting tool: initial findings. Otolaryngol Head Neck Surg 163(1):132-134. https://doi. org/10.1177/0194599820922992

11. Lechien JR, Chiesa-Estomba CM, De Siati DR, Horoi M, Le Bon SD, Rodriguez A, Dequanter D, Blecic S, El Afia F, Distinguin L, Chekkoury-Idrissi Y, Hans S, Delgado IL, Calvo-Henriquez C, Lavigne P, Falanga C, Barillari MR, Cammaroto G, Khalife M, Leich P, Souchay C, Rossi C, Journe F, Hsieh J, Edjlali M, Carlier R, Ris L, Lovato A, De Filippis C, Coppee F, Fakhry N, Ayad T, Saussez S (2020) Olfactory and gustatory dysfunctions as a clinical presentation of mild-to-moderate forms of the coronavirus disease (COVID-19): a multicenter European study. Euro Arch Oto-Rhino-Laryngol 277(8):2251-2261. https://doi.org/10.1007/ s00405-020-05965-1

12. Parma V, Ohla K, Veldhuizen MG, Niv MY, Kelly CE, Bakke AJ, Cooper KW, Bouysset C, Pirastu N, Dibattista M (2020) More 
than smell-COVID-19 is associated with severe impairment of smell, taste, and chemesthesis. Chem Senses 45:609-622. https ://doi.org/10.1093/chemse/bjaa041

13. COVID-19 Guideline of Ministry of Health, Republic of Turkey (2020). https://covid19bilgi.saglik.gov.tr/depo/rehberler/covid -19-rehberi/COVID-19_REHBERI_GENEL_BILGILER_EPIDE MIYOLOJI_VE_TANI.pdf

14. Hopkins C, Surda P, Kumar N (2020) Presentation of new onset anosmia during the COVID-19 pandemic. Rhinology 58(3):295298. https://doi.org/10.4193/Rhin20.116

15. Haehner A, Draf J, Drager S, de With K, Hummel T (2020) Predictive value of sudden olfactory loss in the diagnosis of COVID-19. Orl J Oto- Rhino-Lary 82(4):175-180. https://doi. org/10.1159/000509143

16. Izquierdo-Dominguez A, Rojas-Lechuga MJ, Chiesa-Estomba C, Calvo-Henriquez C, Ninchritz-Becerra E, Soriano-Reixach M, Poletti-Serafini D, Villarreal IM, Maza-Solano JM, MorenoLuna R, Villarroel PP, Mateos-Serrano B, Agudelo D, Valcarcel F, Del Cuvillo A, Santamaria A, Marino-Sanchez F, Aguilar J, Verges P, Inciarte A, Soriano A, Mullol J, Alobid I (2020) Smell and taste dysfunctions in COVID-19 are associated with younger age in ambulatory settings-a multicenter cross-sectional study. J Investig Allergol Clin Immunol. https://doi.org/10.18176/jiaci .0595

17. Klopfenstein T, Kadiane-Oussou NJ, Toko L, Royer PY, Lepiller Q, Gendrin V, Zayet S (2020) Features of anosmia in COVID-19. Med Mal Infect 50(5):436-439. https://doi.org/10.1016/j.medma 1.2020 .04 .006

18. Meini S, Suardi LR, Busoni M, Roberts AT, Fortini A (2020) Olfactory and gustatory dysfunctions in 100 patients hospitalized for COVID-19: sex differences and recovery time in real-life. Eur Arch Oto-Rhino-Laryngol 277(12):3519-3523. https://doi. org/10.1007/s00405-020-06102-8

19. Sayin I, Yasar KK, Yazici ZM (2020) Taste and smell impairment in COVID-19: An AAO-HNS anosmia reporting tool-based comparative study. Otolaryngol Head Neck Surg. https://doi. org/10.1177/0194599820931820

20. Seiden AM (2004) Postviral olfactory loss. Otolaryngol Clin North Am 37(6):1159-1166. https://doi.org/10.1016/j. otc.2004.06.007

21. Vaira LA, Deiana G, Fois AG, Pirina P, Madeddu G, De Vito A, Babudieri S, Petrocelli M, Serra A, Bussu F, Ligas E, Salzano G, De Riu G (2020) Objective evaluation of anosmia and ageusia in COVID-19 patients: single-center experience on 72 cases. Head Neck 42(6):1252-1258. https://doi.org/10.1002/hed.26204

22. Barillari MR, Bastiani L, Lechien JR, Mannelli G, Molteni G, Cantarella G, Coppola N, Costa G, Trecca EMC, Grillo C, La Mantia I, Chiesa-Estomba CM, Vicini C, Saussez S, Nacci A, Cammaroto G (2020) A structural equation model to examine the clinical features of mild-to-moderate COVID-19: a multicenter Italian study. J Med Virol. https://doi.org/10.1002/jmv.26354

23. Mercante G, Ferreli F, De Virgilio A, Gaino F, Di Bari M, Colombo G, Russo E, Costantino A, Pirola F, Cugini G, Malvezzi L, Morenghi E, Azzolini E, Lagioia M, Spriano G (2020) Prevalence of taste and smell dysfunction in coronavirus disease 2019. Jama Otolaryngol 146(8):723-728. https://doi.org/10.1001/jamao to. 2020.1155

24. Netland J, Meyerholz DK, Moore S, Cassell M, Perlman S (2008) Severe acute respiratory syndrome coronavirus infection causes neuronal death in the absence of encephalitis in mice transgenic for human ACE2. J Virol 82(15):7264-7275. https://doi. org/10.1128/JVI.00737-08

25. Vaira LA, Salzano G, Fois AG, Piombino P, De Riu G (2020) Potential pathogenesis of ageusia and anosmia in COVID-19 patients. Int Forum Allergy Rhinol 10(9):1103-1104. https://doi. org/10.1002/alr.22593

26. Brann DH, Tsukahara T, Weinreb C, Lipovsek M, Van den Berge K, Gong B, Chance R, Macaulay IC, Chou HJ, Fletcher RB, Das D, Street K, de Bezieux HR, Choi YG, Risso D, Dudoit S, Purdom E, Mill J, Hachem RA, Matsunami H, Logan DW, Goldstein BJ, Grubb MS, Ngai J, Datta SR (2020) Non-neuronal expression of SARS-CoV-2 entry genes in the olfactory system suggests mechanisms underlying COVID-19-associated anosmia. Sci Adv. https ://doi.org/10.1126/sciadv.abc5801

27. Oliviero A, de Castro F, Coperchini F, Chiovato L, Rotondi M (2020) COVID-19 pulmonary and olfactory dysfunctions: is the chemokine CXCL10 the common denominator? Neuroscientist. https://doi.org/10.1177/1073858420939033

28. Yan CH, Faraji F, Prajapati DP, Boone CE, DeConde AS (2020) Association of chemosensory dysfunction and COVID-19 in patients presenting with influenza-like symptoms. Int Forum Allergy Rhinol 10(7):806-813. https://doi.org/10.1002/alr.22579

29. Speth MM, Singer-Cornelius T, Oberle M, Gengler I, Brockmeier SJ, Sedaghat AR (2020) Mood, anxiety and olfactory dysfunction in COVID-19: evidence of central nervous system involvement? Laryngoscope 130(11):2520-2525. https://doi.org/10.1002/ lary. 28964

30. Henry BM, de Oliveira MHS, Benoit S, Plebani M, Lippi G (2020) Hematologic, biochemical and immune biomarker abnormalities associated with severe illness and mortality in coronavirus disease 2019 (COVID-19): a meta-analysis. Clin Chem Lab Med 58(7):1021-1028. https://doi.org/10.1515/cclm-2020-0369

31. Zhou S, Wang Y, Zhu T, Xia L (2020) CT features of coronavirus disease 2019 (COVID-19) pneumonia in 62 patients in Wuhan, China. Am J Roentgenol 214(6):1287-1294

32. Abdullahi A, Candan SA, Abba MA, Bello AH, Alshehri MA, Afamefuna Victor E, Umar NA, Kundakci B (2020) Neurological and musculoskeletal features of COVID-19: a systematic review and meta-analysis. Frontiers in neurology 11:687

33. Panuganti BA, Jafari A, MacDonald B, DeConde AS (2020) Predicting COVID-19 incidence using anosmia and other COVID-19 symptomatology: preliminary analysis using Google and Twitter. Otolaryngol-Head and Neck Surg 163:491

34. Bendau A, Petzold MB, Pyrkosch L, Maricic LM, Betzler F, Rogoll J, Grosse J, Strohle A, Plag J (2020) Associations between COVID-19 related media consumption and symptoms of anxiety, depression and COVID-19 related fear in the general population in Germany. Eur Arch Psychiatry Clin Neurosci. https://doi. org/10.1007/s00406-020-01171-6

Publisher's Note Springer Nature remains neutral with regard to jurisdictional claims in published maps and institutional affiliations. 